\title{
Energy Efficient Routing with MAX-Min Energy Scheme in Ad-hoc On-Demand Multipath Distance Vector routing for MANET
}

\author{
Harshad Vazar \\ M. Tech Student, \\ LNCT Bhopal, India
}

\author{
Vineet Richarya, Ph.D \\ Head of Computer Science \& \\ Engineering Department, \\ LNCT Bhopal, India
}

\author{
Alekh Dwivedi \\ Asst. Professor,CSE \\ LNCT Bhopal, India
}

\begin{abstract}
During the past few years, there has been increasing interest in the design of energy efficient protocols for wireless ad-hoc networks. Node within an ad hoc network generally relies on batteries (or exhaustive energy sources) for power. Since these energy sources have a limited lifetime, power availability is one of the most important constraints for the operation of the ad hoc network. Therefore energy efficiency is of vital importance in the design of protocols for the application in such networks and efficient operations are critical to enhance the network lifetime. A routing protocol that does not take into account of congestion control will result in usage of paths that are already heavy in traffic load. It will add more burdens on the energy consumption to these paths and indirectly lead to imbalanced energy consumption of the whole network. The nodes in a high traffic load path will 'die' off faster than nodes in paths that have lower traffic load. AOMDV protocol is provided the alternative path from source to destination so that if one path is congested another path can be use to send the packet from source to destination and thus reduce energy consumption. in these research work we are using threshold value to choose some path from the selected path then average energy scheme is used to choose the path for packet delivery.
\end{abstract}

\section{Keywords}

MANET, AOMDV, Energy, Congestion.

\section{INTRODUCTION}

Mobile Ad hoc Network (MANET) is a dynamically reconfigurable wireless network with no fixed infrastructure .each node acts as a router and host and it moves in an arbitrary manner. in many ad hoc networks, each node is powered by a battery and has limited energy supply. Over time, various nodes will deplete their energy supplies and drop out of the network. Unless nodes are replaced or recharged, the network will eventually become partitioned. In a large network, relatively few nodes may be able to communicate directly with their intended destinations. Instead most nodes must rely on other nodes to forward their packets. Some nodes may be especially critical for forwarding these packets because they provide the only path between certain pair of nodes. Associated with each node that depletes its battery and stops operating, there may be number of other nodes that no longer communicate. Energy is scare by the fact that the devices are mobile i.e. they must be small and therefore cannot be fitted with large battery packs. For these reasons a large number of researchers have focused on design of energy efficient routing protocols [5].

\section{ROUTING PROTOCOLS IN MANET}

Routing Protocols in MANET are usually categorized as table-driven and on-demand driven depending upon their network structure, communication model, routing approach and state information but for the most part of these are made depending on routing policy and network structure based on the timing of when the routes are updated.

\subsection{Table-Driven Routing Protocols}

In proactive-routing protocols, each node has preserves one or more routing tables about nodes in the network. This routing protocol updates the routing table information either periodically or in response to change in the network topology. In this protocol source node does not need routediscovery procedures to find a destination node. On the other hand protocols is maintaining a reliable and updating routing table requires considerable messaging overhead, which consumes power and bandwidth ,and decreases throughput ,especially in the case of a large number of high mobility of node. Table-driven protocols are DSDV, WRP, FSR, OLSR, CGSR and TBRPF etc.

\subsection{On-Demand Routing Protocols}

The benefits of these protocols are that overhead messaging is reduced. One of the disadvantages of these protocols is the delay in discovering a new route. The different types of reactive routing protocols are: Dynamic Source Routing, Adhoc On-demand Distance Vector Routing, Ad hoc Ondemand multi-path distance vector routing (AOMDV) and temporally Ordered Routing Algorithm (TORA).

On-demand routing protocols is an initialization of a route discovery method by the source node to find the route to the destination node when the source node has to send data packets. When a route is establish, the route maintenance is initiated to maintain transmission and this route until it is no longer required or the destination is not reachable. These protocols are reduced overhead messages and delay in discovering a new route. On-demand protocols are DSR, AODV, AOMDV, TOTA, [6] etc.

\section{BACKGROUND}

On demand routing protocols work on the principle of creating routes as and when required between a source and destination node pair in a network topology: 


\subsection{Ad-hoc On-Demand Distance Vector Routing (AODV)}

AODV is a reactive protocol that discovers route on an as needed basis using a route discovery mechanism. AODV uses traditional routing tables with one entry per destination. without using source routing,AODV relies on its routing table entries to propagate an RREP (Route Reply) back to the source and also to route data packets to the destination.AODV uses sequence numbers maintain at each destination to determine freshness of routing information and to prevent routing loop. All routing packets carry these sequence numbers.

AODV maintains timer-based states in each node, for utilizations of individual routing table entries, whereby older unused entries are removed from the table.

Predecessor node sets are maintained for each routing table entry, indicating the neighboring nodes sets which use that entry to route packets. These nodes are notified with RERR (Route Error) packets when the next-hop link breaks. These packets get forwarded by each predecessor node to its predecessors, effectively erasing all routes using the broken link. Route error propagation in AODV can be visualized conceptually as a tree who's root is the node at the point of failure and all sources using the failed link as the leaves. The advantage of AODV are that less memory space is required as a information of only active routes are maintained, in turn increasing the performance, while the disadvantage is that this protocol is not scalable and in large networks it does not perform well and does not support asymmetric links.

\subsection{Ad-hoc On-demand Multipath Distance Vector Routing (AOMDV)}

Ad-hoc On-demand Multipath Distance Vector Routing (AOMDV) protocol is an extension to the AODV protocol for computing multiple loop-free and link disjoint paths. The routing entries for each destination contain a list of the nexthops along with the corresponding hop counts. All the next hops have the same sequence number. This helps in keeping track of a route. For each destination, a node maintains the advertised hop count, which is defined as the maximum hop count for all the paths, which is used for sending route advertisements of the destination. Each duplicate route advertisement received by a node defines an alternate path to the destination. Loop freedom is assured for a node by accepting alternate paths to destination if it has a less hop count than the advertised hop count for that destination. Because the maximum hop count is used, the advertised hop count therefore does not change for the same sequence number. When a route advertisement is received for a destination with a greater sequence number, the next-hop list and the advertised hop count are reinitialized. AOMDV can be used to find node-disjoint or link-disjoint routes. To find node-disjoint routes, each node does not immediately reject duplicate RREQs. Each RREQs arriving via a different neighbor of the source defines a node-disjoint path. This is because nodes cannot be broadcast duplicate RREQs, so any two RREQs arriving at an intermediate node via a different neighbor of the source could not have traversed the same node. In an attempt to get multiple link-disjoint routes, the destination replies to duplicate RREQs, the destination only replies to RREQs arriving via unique neighbors. After the first hop, the RREPs follow the reverse paths, which are node disjoint and thus link-disjoint. The trajectories of each RREP may intersect at an intermediate node, but each takes a different reverse path to the source to ensure link disjointness. The advantage of using AOMDV is that it allows intermediate nodes to reply to RREQs, while still selecting disjoint paths. But, AOMDV has more message overheads during route discovery due to increased flooding and since it is a multipath routing protocol, the destination replies to the multiple RREQs those results are in longer overhead [7].

\section{TRAFFIC patterns}

Traffic patterns describe how the data is transmitted from source to destination. The two types of traffic patterns employed in MANET are CBR and TCP traffic patterns.

\subsection{CBR Traffic Pattern}

The qualities of Constant Bit Rate (CBR) traffic pattern are i) unreliable: since it has no connection establishment phase, there is no guarantee that the data is transmitted to the destination. ii) Unidirectional: there will be no acknowledgment from destination for confirming the data transmission and iii) predictable: fixed packet size, fixed interval between packets, and fixed stream duration.

\subsection{TCP Traffic Pattern}

The qualities of transmission control protocol (TCP) traffic are i) reliable: since connection is established prior to transmitting data, there is a guarantee that the data is being transmitted to the destination, ii) bi-directional: every packet that has to be transmitted by the source is acknowledged by the destination , and iii) conformity: there will be flow control of data to avoid overloading the destination and congestion control exists to shape the traffic such that it conforms to the available network capacity. Today more than $95 \%$ of the Internet protocol traffic is carried out through TCP [8].

\section{RELATED WORK}

In this research [11] paper, all nodes that are used frequently along discovered paths to provide connectivity for Longer durations may lead to a more graceful degradation of the network resulting in network partitioning. To help solve the problem of network partitioning, we therefore proposed an energy optimization based path selection algorithm for the IEEE 802.11s HWMP. The proposed algorithm will result in an unbiased energy spending among the nodes which also maximizes the network lifespan. When given multiple paths to choose from during the routing process, choose the path that will help prolong the network lifetime. In order to ensure that networks with low-energy nodes are kept alive. The HWMP protocol does not use the one good path it has discovered for every transmission; instead a set of good paths are kept in its routing table and one good path is chosen among the many available paths based on a probabilistic fashion. Therefore, different paths are used at different times instead of using the single path for all the transmissions in the network and in return energy depletion in certain nodes is avoided to avoid network portioning.

In this research [13] paper, a number of energy aware routing protocols and discussed their effects on various QoS parameters intrinsic to traditional networks such as delay, bandwidth, packet loss and parameters more specific to a mobile ad hoc environment such as energy consumption, overall network lifetime, mobility etc. The study showed that energy aware protocols usually increase the overall functioning lifetime of the network by efficiently using every node's energy resources. Every energy aware routing 
protocol performs well in terms of data delivery as inferred from low packet loss. Most applications require reliable data transfer between them and wireless medium is known to be unreliable. Unreliable wireless links have a negative effect on energy savings as repeated retransmissions or route discoveries may occur.

\section{PROPOSED WORK}

In our proposed work we proposed MAX-Min energy aware scheme that gives better performance of the network. For MAX-Min energy scheme implementation we use routing protocol as AOMDV that gives multiple paths between sender to receiver and after retrieving multiple paths we select MAXIMUM energy base route. That best energy bases path gives maximum reliable as compare to existing route.

Step 1: setup initial node position (Xi, Yi) for all node

$$
\text { //Coordinate Value }
$$

Step 2: Set Source = S;

// List of sources

Step 3: Set Destination $=\mathrm{D}$

\section{// List of Destinations}

Step 4: Set Routing Protocol $=$ AOMDV

// for load distribution of intermediate nodes

Step 5: Configure initial energy of nodes $=\mathrm{E}$

Step 6: MAC protocol = MAC 802.11

Step 7: Broadcast-Route(S, D, E)

Step 8: filtering out the route who's any of the node have energy value less than the threshold value and keep the other route.

Step 9: Now we apply Max-min energy scheme before selecting any path based on average energy value based scheme.

Step 10: send the packets using primary optimum selected

Path.

Power consumption in MANETs is an important issue because all or most of the nodes are battery supplied, and the communication infrastructure is composed of the same nodes which are using it. In such context, optimizing energy consumption also means maximizing the overall usability of the network. Power is required for both processing (e.g. protocols operations and applications execution) and communication (e.g. control and data messages transmission).

\section{SIMULATION AND RESULT}

Network Simulator 2.35 was used for the simulation purpose.

\subsection{Performance Parameter}

The different parameters considered for simulation are listed as follows:

Packet Delivery Ratio: This is the ration of the data packets delivered to the destination to those generated by the traffic source.

Routing Overhead: Routing overhead is the number of routing packets transmitted per data packet delivered to the destination

Throughput: It is the average rate of successful message delivery over the communication channel.

\subsection{Simulation Parameter}

Our simulation setting and parameters are summarized in Table1.

Table 1: Simulation parameter

\begin{tabular}{|l|l|}
\hline Simulator Used & NS2.35 \\
\hline Number of nodes & 30 \\
\hline Dimension of simulated area & $800 \mathrm{~m} \times 600 \mathrm{~m}$ \\
\hline Routing Protocol & AOMDV \\
\hline Simulation time & 100 Seconds \\
\hline Transport Layer & TCP ,UDP \\
\hline MAC Type & Mac 802.11 \\
\hline Traffic type & CBR,FTP \\
\hline Packet size & 512 Bytes \\
\hline Number of traffic connections & 4 \\
\hline Maximum Speed (m/s) & Random \\
\hline Transmission Range & $250 \mathrm{~m}$ \\
\hline Transmission Energy & 1.5 Joule \\
\hline Receiving Energy & 1 Joule \\
\hline
\end{tabular}

\subsection{Result}

In our case PDF is best that means our performance is very good, in Figure 1, we shows packet delivery ratio at the time of old scheme and proposed AOMDV scheme and get proposed case nearly better as compare to existing system that is nearly 98.16 percentages. 


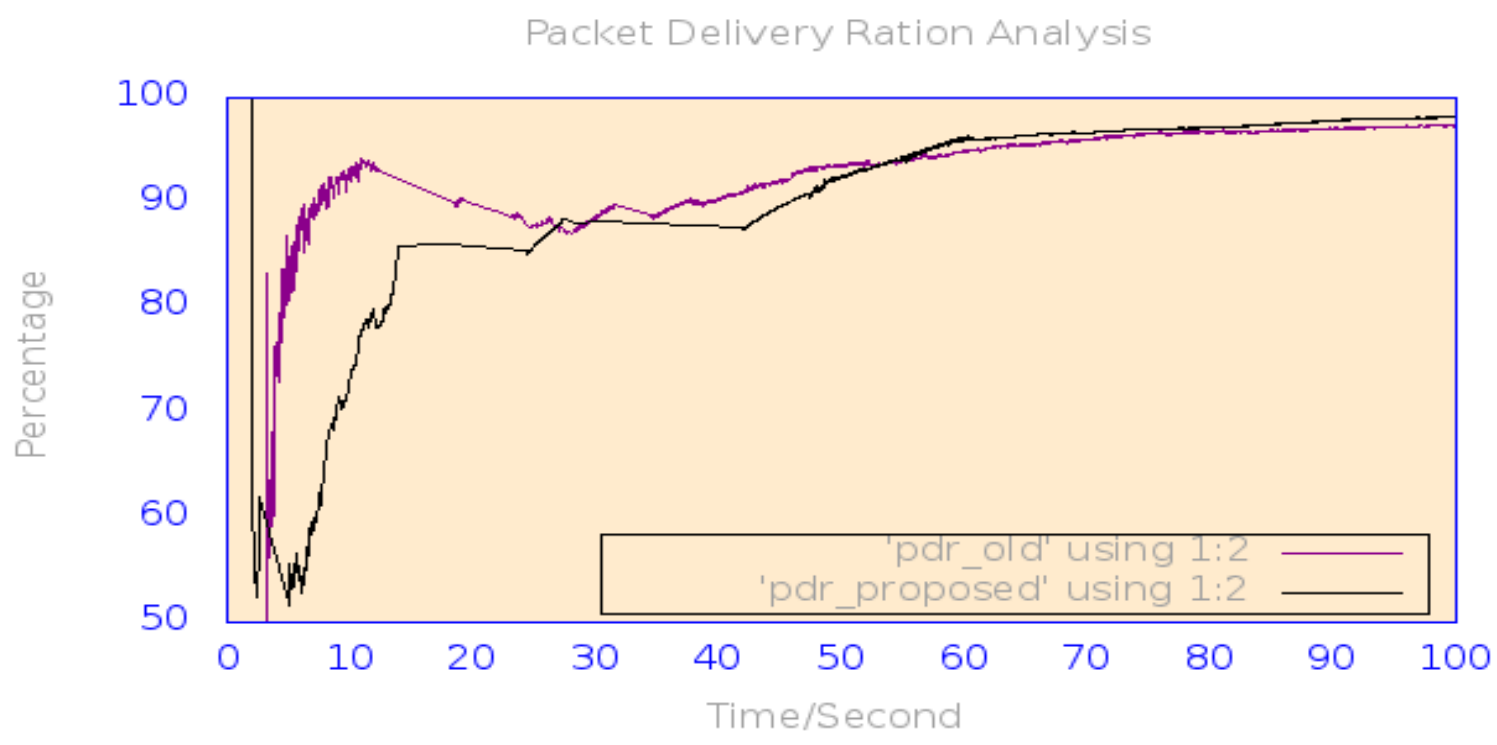

Figure 1: PDR comparison between old and proposed Case.

Routing message overhead is calculated as the total number of control packets transmitted. The increase in the routing message overhead reduces the performance of the ad-hoc network as it consumes portions from the bandwidth available to transfer data between the nodes.

Here we analyze routing packets in old case as well as proposed case and in old case routing overhead is nearby 3698 at the end of simulation and in proposed AOMDV case routing packets is nearly 3504 , according to Figure 2, our routing over head increased proportional to simulation time increases that conclude overhead increased if simulation time increases Routing message overhead is calculated as the total number of control packets transmitted. The increase in the routing message overhead reduces the performance of the adhoc network as it consumes portions from the bandwidth available to transfer data between the nodes.

Here we analyze routing packets in old case as well as proposed case and in old case routing overhead is nearby 3698 at the end of simulation and in proposed AOMDV case routing packets is nearly 3504 , according to Figure 2, our routing over head increased proportional to simulation time increases that conclude overhead increased if simulation time increases.

\section{Routing Load Analysis}

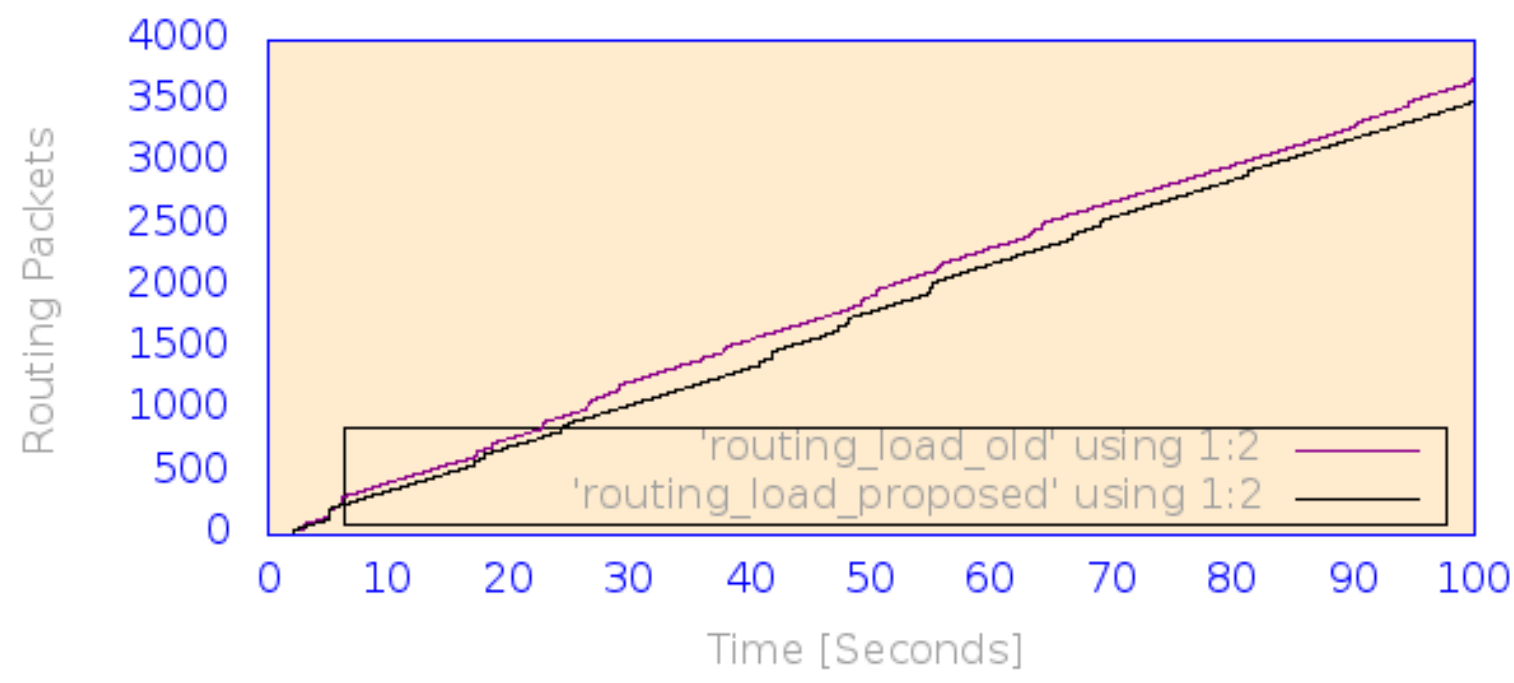

Figure 2: Routing Overhead comparison between old and proposed case. 


\section{CONCLUSION}

Due to the limited battery capacity the number of mobile nodes are exhausted rapidly by that the sender are not communicate with receiver and also intermediate nodes are not communicate with neighbor. Energy is a main challenge in Mobile Ad hoc Network (MANET). We have presented the state-of-the-art of TCP over mobile Ad hoc networks. Table 2 shows that the proposed case is better with all the above mentioned parameter.

Table 2: Overall Analysis

\begin{tabular}{|l|l|l|}
\hline \multicolumn{3}{|c|}{ Overall Analysis } \\
\hline PARAMETER & Old & Proposed \\
\hline ROUTING PKTS & 3698 & 3504 \\
\hline PDF & 97.28 & 98.16 \\
\hline NRL & 1.14 & 1.03 \\
\hline DROP RTS & 12 & 3 \\
\hline No. of Dropped Data & 91 & 64 \\
\hline Actual Performance & $97.56 \%$ & $99.14 \%$ \\
\hline
\end{tabular}

Future work we try to implement to same technique in Worldwide Interoperability for Microwave Access (WIMAX) technology and measure performance of parameter in mobile ad-hoc network.

\section{REFERENCES}

[1] Bhabani Sankar Gouda, Arkaprava Bhaduri Mandal, K. Laxmi Narayan "Simulation and comparative analysis of energy conservation performance metric for ERAODV , AODV and DSDV ROUTING PROTOCOL in MANET”, IEEE India ,2012.

[2] Anu Kumari, Arvind Kumar, Akhil Sharma "Survey Paper on Energy Efficient Routing Protocol in MANET”,in IJARCSSE, vol. 3,2013.

[3] R Gupta, A A.Waoo, S Sharma and P. S Patheja "A Research Paper on Comparison between Energy Efficient Routing Protocol with Energy and Location in MANET “ IOSR-JCE,vol. 9,2013.

[4] Wei Liu,Chi Zhang, Guoliang Yao, Yuguang Fang "DELAR: A Device-Energy-Load Aware Relaying
Framework for Heterogeneous Mobile Ad Hoc Networks",IEEE,vol. 29,2011.

[5] GETSY S SARA, NEELAVATHY PARI. S, SRIDHARAN.D "Energy Efficient Ad Hoc On Demand Multipath Distance Vector Routing Protocol" ,IJRTE,vol. 2,2009.

[6] Bhabani Sankar Gouda, Chandan Kumar Behera, Ranjit Kumar Behera "A Scenario Based Simulation Analysis and Performance Evaluation of Energy Efficiency Enhancement of Routing Protocols in MANET" ,IEEE,2013

[7] S. R. Biradar, Koushik Majumder, Subir Kumar Sarkar, Puttamadappa C "Performance Evaluation and Comparison of AODV and AOMDV", IJCSE, vol. 3 ,2010.

[8] Avinash Giri, Jitendra Prithviraj ,Aashok verma "Analysis of unipath and multipath routing protocols in mobile adhoc networks", IJSSAN,vol. 2,2012.

[9] Mahesh K. Marina, Samir R. Das "Ad hoc on-demand multipath distance vector routing", Wiley InterScience, 2006.

[10] Martin M. Mhlanga, Thomas O. Olwal, Murimo B Mutanga, Mathew O. Adigun "Energy Optimization based Path Selection Algorithm for IEEE 802.11s Wireless Mesh Networks", IEEE, 2011.

[11] Mahesh K Marina, Samir R Das "On-demand Multipath Distance Vector Routing in Ad Hoc Networks", IEEE, 2001.

[12] Charu, Vivek Arya "A Quality of Service Analysis of Energy Aware Routing Protocols in Mobile Ad Hoc Networks",IEEE, 2013.

[13] Prashant Kumar Maurya1, Gaurav Sharma, Vaishali Sahu, Ashish Roberts, Mahendra Srivastava "An Overview of AODV Routing Protocol ", IJMER, vol. 2, 2012

[14] P Sivakumar ,Dr. K Duraiswamy “ A QoS Protocols for mobile Ad Hoc Netwroks based on the Load Distribution”, IEEE,2010. 